\title{
Quantitative Structural Analysis of Complex Materials by Scanning Nanobeam Diffraction
}

\author{
Christoph Gammer ${ }^{1,2}$, Burak V. Özdöl ${ }^{1}$, Karen C. Bustillo ${ }^{1}$, J. Ciston ${ }^{1}$ and Andrew M. Minor ${ }^{1,3}$ \\ 1. National Center for Electron Microscopy, Molecular Foundry, Lawrence Berkeley National \\ Laboratory, Berkeley, CA, USA. \\ 2. Physics of Nanostructured Materials, Faculty of Physics, University of Vienna, Austria. \\ 3. Department of Materials Science and Engineering, University of California, Berkeley, CA, USA.
}

Conventional TEM is routinely used to study structural properties of materials with nanometer resolution by using diffraction contrast imaging techniques. In addition electron diffraction can be used to acquire focused local information on the orientation or crystal structure. Alternatively, ADF-STEM uses a converged scanning beam and an annular detector and can also be used to image defects using diffraction contrast [1]. In both techniques the possible images that can be formed are limited by the physical shapes and sizes of the apertures or detectors.

The present work shows that acquiring a diffraction pattern for each beam position in STEM can overcome these limitations. STEM diffraction mapping yields a rich dataset that can be analyzed and presented in multiple ways after the experiment [2]. One practical limitation is the speed of the electron detector. In this work a Gatan K2 IS direct detection camera operating at a frame rate of $400 \mathrm{f} / \mathrm{s}$ is used to record a map, consisting of more than 65,500 diffraction patterns, in only 2.7 minutes. To evaluate the large dataset, custom DigitalMicrograph scripts were written. These new diffraction mapping techniques are applied to a precipitation strengthened ferritic alloy. Figure 1 shows the experimental setup for STEM diffraction mapping. During acquisition of an ADF-STEM image (cf. Fig. 1a) a diffraction pattern is recorded for every probe position. The resulting series of diffraction patterns can be used to reconstruct a dark-field image by calculating the mean intensity for the virtual aperture at each probe position. Figure $1 \mathrm{~b}$ shows a virtual dark-field image generated by selecting a superlattice reflection of the ferritic alloy. The image reveals the ordered precipitates in the alloy. Similarly, a virtual selected area diffraction pattern can be formed by placing a virtual aperture anywhere on the sample. The respective diffraction pattern is calculated by summing all diffraction patterns from that region. Figure 1c shows the diffraction pattern corresponding to an ordered precipitate. The major advantage is that arbitrarily shaped virtual apertures can be specifically designed according to the underlying crystal structure.

The stack of diffraction patterns acquired by STEM diffraction mapping can not only be used for phase or orientation mapping but can also generate strain maps. The strain is calculated from the shift of the diffracted peaks (cf. Fig. 1d). The positions of the diffracted peaks are deduced to sub-pixel accuracy using template matching [3]. STEM diffraction mapping is robust against sample drift and does not rely on a field of view with a single well-aligned zone axis. Therefore, it is well suited to measure nanoscale strain in highly defected materials, where strain mapping techniques based on high-resolution or diffraction contrast would fail. It will be demonstrated that STEM diffraction mapping can also be combined with in-situ microscopy as ultrafast electron detectors enable real-time collection of diffraction maps at sufficient speeds to carry out diffraction mapping during continuous in-situ deformation [4]. 


\section{References:}

[1] P. Phillips, et al, Ultramicroscopy 111 (2011), p. 1483.

[2] C. Gammer, et al, Ultramicroscopy 155 (2015), p. 1.

[3] V.B. Ozdol, et al, Applied Physics Letters 106 (2015), p. 253107.

[4] The authors acknowledge support by the Austrian Science Fund (FWF):[J3397] and the Molecular Foundry, Lawrence Berkeley National Laboratory, which is supported by the U.S. Dept. of Energy under Contract \# DE-AC02-05CH11231.

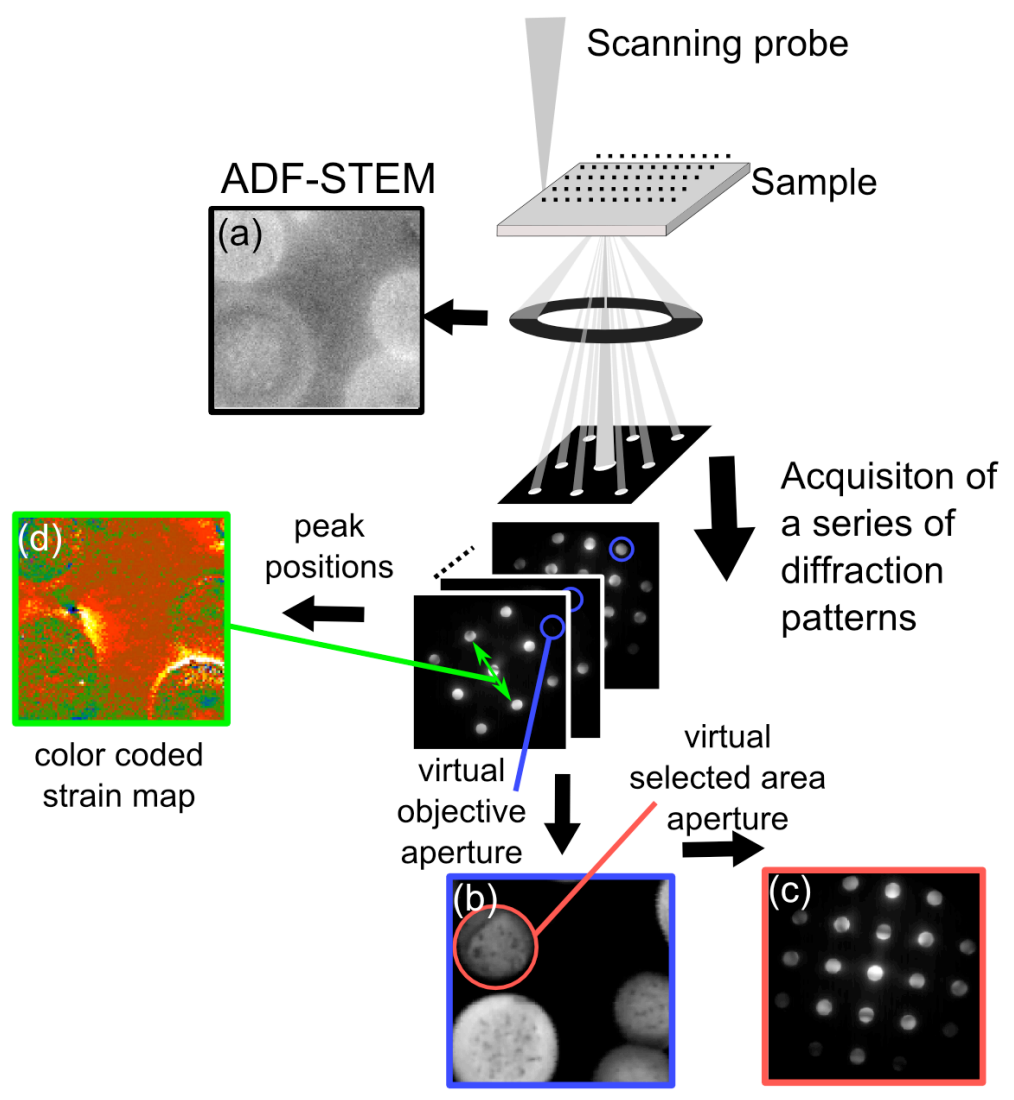

Figure 1. STEM diffraction mapping: (a) During acquisition of an ADF-STEM image, a diffraction pattern is recorded for every probe position. (b) A virtual dark-field image is reconstructed by calculating the mean intensity in a virtual objective aperture (blue circle) for every probe position. (c) A virtual selected area aperture diffraction pattern is generated by selecting an arbitrary region in the image (red circle) and summing all corresponding diffraction patterns. (d) A strain map can be generated by detecting the positions of the diffracted peaks in each diffraction pattern. 